\title{
Getting serious about the dirty little secrets of health care reform to guarantee true Universal Health Care for all Filipinos
}

Republic Act 11223, known as the Universal Health Care (UHC) Act, has a triple goal of providing quality healthcare services that are equitable, accessible and cost-effective for all Filipinos. At long last, no longer would health care reform have to be re-invented as new DOH Administrative Orders or Executive Orders, which changes not only with every new Presidency, but in fact with every new Secretary of Health (recall the "Integrated Public Health System of 1983", "Health Sector Reform Agenda of 1999", the "FOURmula ONE for Health of 2005", the "FOURmula ONE Plus for Health of 2019" programs). Finally, we now have a law that mandates lasting health care reform which are multi-administration in length, and multi-sectoral in scope. With this new law, however, we now have to accept the dirty little secrets that all past health care reform programs have learned. That medical science alone is not enough to bring about lasting improvements in the mortalities and morbidities for every Filipino man, woman, and child. That every new advancement in medicine, vaccination, therapy, or diagnostics, have actually been met with: new schemes for fraud; new opportunities for program failures due to increasing infrastructure demands including more electricity, more expensive machines, faster internet access, and others; and new healthcare workers who become overworked, under-compensated, and even under-protected from politicians and patients who attack the new advances due to ignorance or unfounded fears.

As the old adage goes, the correct naming of a problem is $50 \%$ of the solution. Fortunately, just as the dirty little secret have metamorphosed with each medical science improvement, so have Information Technology, Behavior Science, Management Science, Economic Evaluation science, and Criminology also improved. Thus, the modern Evidence-Based Policy (EBP) development must actively supplement medical science with these other sciences to ensure that the promise of better, safer, and more cost-effective improvements in mortality and morbidity is actually enjoyed by all beneficiaries.

As the main steward of health, the Department of Health (DOH) takes the lead in detailing the operationalization of the Law, hence, formulating the prescribed provisions of the Implementing Rules and Regulations (IRR). Because we need to ensure the success of this Law, it is imperative for $\mathrm{DOH}$ to use evidence-based policy tools and papers, and to yield consensus recommendations from all involved stakeholders. Hence, the UP Manila Health Policy Development Hub, having the special niche as a multi-science policy research group, was granted the AHEAD-HPSR funding to generate evidences as inputs in crafting the UHC Act IRR.

The 15 policy papers in this issue formed part of the expected products from a research grant on "Development of Policy Notes or Evidence Summaries" submitted by the University of the Philippines Manila Health Policy Development Hub (UPM HPDH), otherwise known as Policy Hub, to the AHEAD-HPSR program of DOH in 2018. Created in 2015, UPM HPDH is a think tank and network of health policy advocates, analysts, and multidisciplinary researchers under the Office of the Chancellor of the University of the Philippines Manila and aims to be a national leader in formulating policy statements based on empirical, unbiased data, and appropriate statistical and policy analysis, to aid in the implementation of policy in different local settings. Since its establishment, the Policy Hub has conducted policy round table discussions on high impact and controversial health policies, provided trainings for policy makers and students, held special mentoring sessions with policy analysts, formed technical working groups for policy analysis that included critical assessment of evidence, dissemination and publication, among others. With the ubiquitous presence of fake news and other unreliable health information accessible to the public in the internet and social media, the Policy Hub aims to provide reliable and evidence-based health policy analyses and statements as an act of responsive public service and advocacy.

This special issue of the journal contains position papers and policy statements that generated evidence summaries and consensus recommendations as inputs for the IRR of the UHC Act of 2020. These 15 policy papers focus on salient features of the UHC Act that revolve on the following: addressing primary care inequities; contracting out of health services for province-level integration of healthcare system; determining hospital bed capacity; gaps and gray areas identified in the IRR; criteria for population versus Individual-based health services; rationalizing health personnel financing schemes; third party accreditation; equitable health investment; return service agreement; and, include vital health concerns drawn from other health-related laws such as mental health and oral health that are integral part of the healthcare services in the UHC Act. 
Producing evidence-based data and information in aid of legislation is evolving as a standard in developing sound health policy and decision. ${ }^{1}$ We all know the power that a state-sponsored health policy exudes. It significantly influences how health is distributed, accessed, and acquired. Thus, a health policy evidenced by the sciences, if deployed properly with strong political will, certainly leads to healthy and happy citizens.

The policy papers in this special issue embody a collaboration between UP Manila and DOH. A collaboration that could serve as a model wherein a health sciences academic institution and a national health agency of the government worked in synergy towards a public health policy that balances population health, scientific evidences, and fiscal and risk management. This was the first formal application of evidence-based policy (EBP) development between DOH, UP Manila, and stakeholders for evidence-informed and unbiased policy making.

Evidence-based policy (EBP) development "refers to an approach that levers the best available objective evidence from research to identify and understand issues so that policies can be crafted by decision makers that will deliver desired outcomes effectively, with a minimal margin of error and reduced risk of unintended consequences." ${ }^{2}$ The rationale therefore for EBP, is to advocate a more systematic approach that is both rigorous and encompassing to inform the policy process. ${ }^{3}$ Its objective consequently is not to produce the solutions; rather it is to utilize the best evidences, to provide credible information and analysis as a tool in policy making. ${ }^{2}$ As it uses these clear, unbiased and objective EBP policies, front line implementers can better contextualize, augment and deliver the intended health and health related improvements, by reducing uncertainty, increasing consistency, and providing accountability.

We assert that the 15 policy papers contained in this special issue are products of EBP development and were meant to produce grounded data and information from the best scientific evidences and actual stakeholder experiences, that minimize and even prevent fraud, program failures, and worker fatigue and demoralization, so that we will finally attain true universal health care for all Filipinos in all stages and walks of life.

\section{Leonardo R. Estacio, Jr., MCD, MPH, PhD Dean College of Arts and Sciences University of the Philippines Manila}

\author{
Hilton Y. Lam, MHA, PhD \\ Director \\ Institute of Health Policy and Development Studies \\ National Institutes of Health \\ University of the Philippines Manila
}

\section{REFERENCES}

1. Brownson RC, Chriqui JF, Stamatakis KA. Understanding evidence-based public health policy. Am J Public Health. 2009 Sep; 99(9):1576-83. doi:10.2105/AJPH.2008.156224.

2. Townsend T, Kunimoto B. Capacity, Collaboration and Culture: The Future of the Policy Research Function in the Government of Canada [Internet]. 2014 [cited 2020 Nov]. Available from:

3. Sutcliffe S, Court J. Evidence-Based Policy Making. Overseas Development Institute. November 2005 [cited 2020 Nov]. Available from: https://www. odi.org/sites/odi.org.uk/files/odi-assets/publications-opinion- files/3683.pdf 